\title{
Violencia sexual infantil y adolescente en la prensa costarricense (2014-2015)
}

\section{Child and adolescent sexual violence in the Costa Rican press (2014-2015)}

\author{
José David Ramírez Roldán* \\ *Universidad de Costa Rica, Costa Rica. Contacto: jdr_r@hotmail.com
}

Referencia/ reference:

Ramírez, J. (2020). Violencia sexual infantil y adolescente en la prensa costarricense (2014-2015). Yulök Revista de Innovación Académica, 4(1), 90-99

\section{Resumen}

Este artículo explora las producciones discursivas emitidas por el periódico La Nación acerca de los factores vinculados a la violencia sexual infantil y adolescente, entre el 1 de enero del 2014 al 23 de abril del 2015. Desde estas perspectivas emitidas por los medios de comunicación escrita se plantea su posición como parte de un sistema de representaciones que circulan en la sociedad civil, partiendo así que, en algunos fenómenos se puede realizar una elaboración sistematizada de los contenidos y reconstruirse indicios estadísticos con una validez relevante de casos para la esfera nacional.

Palabras clave: medios de comunicación de masas, prensa, abuso infantil y adolescente, estadísticas de la comunicación, derecho de la familia.

\section{Abstract}

This paper explores the discursive productions issued by the newspaper La Nación about the factors linked to sexual violence during childhood and adolescence, from January 1, 2014 to April 23, 2015. From these perspectives issued by the media written communication poses its position as part of a system of representations that circulate in civil society, starting in some phenomena can be made a systematic elaboration of the contents and reconstructed statistical evidence with a relevant validity of cases for the national sphere.

Keywords: Mass media, press, Child abuse, Communication statistics, Family law. 
La presente investigación se enfoca en caracterizar y contextualizar los casos de violencia sexual contra menores de edad abordados en la prensa costarricense, utilizando para ello, el periódico La Nación, con una limitación temporal de emisión, entre el 1 de enero del 2014 al 23 de abril del 2015. Asimismo, constantemente se harán algunas reflexiones sobre la posible relación del pasado histórico de este fenómeno sexual en Costa Rica; presentándose como una reseña breve de los antecedentes históricos más recientes de la violación y el abuso sexual en menores de edad en nuestro país, ofreciéndose información útil relacionada con dicha materia. Por consiguiente, habrá que preguntarse: ¡cómo aborda la prensa costarricense los hechos que expresan violencia sexual en menores de edad, desde el periódico La Nación, entre los años $2014-2015 ?$

Empero, se plantea la necesidad de obtener instrumentos reflexivos para analizar con seriedad el tema de la violencia sexual hacia la población menor de edad, mediante el Análisis Crítico del Discurso (ACD). Sin embargo, en este debate es conveniente ampliar el campo de la violencia sexual, desde la aparición del fenómeno en el ámbito familiar hasta la impunidad legal en su conjunto, pues es en el seno familiar donde surge una dinámica interrelacional compleja, y es el lugar por excelencia en el que brota el germen de la pulsión agresiva, siendo esta noción originalmente planteada por la psicóloga Regina Bayo-Borrás (2000, p. 245). Además, se hace referencia, específica, a la correlación matriz de configuración en las relaciones y los vínculos interpersonales durante la infancia o la adolescencia.

Por consiguiente, es evidente que la violencia sexual en menores existe, tanto endógena como exógenamente del ámbito familiar. Los datos del día con día lo confirman, los profesionales en salud, leyes, comunicación y algunas personas que viven de manera cercana a este fenómeno lo comprueban a diario. Asimismo, hay rastros importantes en las páginas de los periódicos, los programas televisivos y las campañas de prevención que pueden revelar parte de la magnitud de la situación. Por ende, en el presente trabajo se escogió analizar 50 notas periodísticas que traten solamente los sucesos nacionales relacionados con el tema. Las noticias se dividirán según dos indicadores de la siguiente forma: 28 notas periodísticas tratan la temática de la violencia sexual, no en forma de casos, sino en discusiones de cómo está actuando el país sobre esa materia; las otras 22 tratan casos específicos de violencia sexual, como abusos, violaciones, entre otros. Aquí es importante aclarar que una noticia se repite para ambos indicadores esto por su riqueza como fuente, tanto contextual como de caso.

Los datos fueron recolectados vía virtual y en distintas secciones de la prensa que presentaron temáticas relacionadas con la violencia sexual contra menores de edad (específicamente, dentro del territorio costarricense o relacionados con este). Aunque bien, esta investigación presenta una limitación para entender con mayor profundidad el tema propuesto, ya que utiliza una sola fuente primaria, el periódico La Nación; dicho recurso ofrece y agiliza datos que nos pueden ayudar a entender los aspectos que generalmente se repiten en el fenómeno de la violencia sexual en menores de edad, en donde se observa la expresión tanto comunicativa como interactiva, dadas en sus respectivos contextos lingüísticos, sociales, políticos, históricos y culturales.

Según la hipótesis de este trabajo, puede existir una latente relación en los discursos informativos dados en la prensa costarricense sobre la violencia sexual infringida a menores de edad y las representaciones de los actores, eventos y problemáticas en disputa. También se propone la existencia de cuatro factores fundamentales que propician y son causa del maltrato y de la violencia sexual en la población menor de edad: a) la posición que ocupan en la sociedad, dependencia del grupo que perpetra el abuso y el maltrato, entre más jóvenes son físicamente más pequeños y menos fuertes, siendo obligados a respetar y obedecer la autoridad del adulto; b) el aislamiento del menor, dentro de la concepción de la familia nuclear cerrada, facilita la agresión, así como las creencias culturales de que los hijos son propiedad de los padres.

El objetivo general es realizar un Análisis Crítico del Discurso de 50 noticias del periódico costarricense La Nación, entre los años 2014 y 2015, a propósito de acontecimientos con presencia de factores vinculados con la violencia sexual infantil y adolescente en Costa Rica. En relación con los objetivos específicos, esta investigación se lleva a cabo con dos: el primero es abordar el contexto de los hechos expuestos por las noticias sobre la supuesta violencia sexual infantil y adolescente, y el segundo, es analizar de forma cuantitativa los recursos y estrategias lingüísticas presentes en el discurso de las noticias del periódico La Nación, acerca de la presencia de factores vinculados con la violación y abuso sexual en menores de edad, para mostrar la construcción de los actores sociales en conflicto. 
Metodológicamente, se realiza un Análisis Crítico de Discurso en las distintas secciones del periódico $\mathrm{La} \mathrm{Na}$ ción, entre el 1 de enero del 2014 al 23 de abril del 2015, que hablen del tema de la violencia sexual en menores de edad. El investigador de este trabajo es consciente de que, por ninguna forma, hay que representar apresuradamente los posibles procesos discursivos que no tengan estrictamente relación entre sí pero que, para fines de la investigación, habrá que tratar de ofrecer una indagación del indicio e hilo conductor de la problemática en estudio y su trato dado en la prensa costarricense, en donde se sugiere, únicamente, la existencia de algún tipo de vínculo.

El sociolingüista Allan Bell en su libro The language of news media "(...) enumera una serie de razones por las cuales los investigadores de diversos ámbitos han estudiado el discurso de los medios" (Vallejo, 2005, p. 8). $\mathrm{Y}$ en este trabajo se señala solo tres útiles maneras con el fin de observar cómo la realización de un ACD de los medios permite la aproximación a sus funciones sociales, por lo que en este escrito se citan las siguientes tres ideas de análisis:

Lo que expresa el discurso sobre la estructura y valores de los medios. Ver los medios como espejo de la más amplia sociedad y cultura inmersa. Atender en efecto de los medios en las actitudes y opiniones de la sociedad, por medio de las representaciones de la gente y los temas (Vallejo, 2005, p. 8).

A nivel metodológico, el Análisis del Discurso aporta una fácil sistematización que no está presente en la mayoría de las aproximaciones a la hora de estudiar las noticias de la prensa. Por ende, permite ir más allá de simples descripciones (de sentido común), tampoco se indican, solamente, hechos de manifestaciones de abusos o violaciones sexuales a menores presentes en los textos de prensa, si no, más bien, se evidencian los mecanismos o las posibles estrategias que se logran construir discursivamente en las representaciones de menores de edad violentados sexualmente, o sea, la analogía de la violencia de unos sobre otros.

\section{Contexto de los hechos expuestos por las no- ticias}

Las noticias que acercan a la situación nacional poseen, en su mayoría, un lenguaje jurídico, por dicha razón, es importante situar el inicio del período de estudio desde “(...) la aprobación de la legislación nacional de mayor importancia en el tema de los derechos de las personas menores de edad" (Martin, s.f.) en Costa Rica. El Código de la Niñez y Adolescencia de Costa Rica, aprobado por la Asamblea General de Costa Rica, fue creado por medio de la Ley N. ${ }^{\circ} 7739$ y fue publicado en La Gaceta N. ${ }^{\circ} 26$, el 6 de febrero de 1998. Este código se fue actualizando con adiciones, reformas y anulaciones hasta convertirse en su última versión dada en enero del 2013. El artículo 58 es el que está más ligado a esta investigación, ya que en la sección de "Políticas nacionales", en el apartado con la letra $f$, se señala que el Estado deberá "propiciar (...) temas relacionados con la educación sexual, la reproducción, el embarazo en adolescentes, las drogas, la violencia de género, las enfermedades de transmisión sexual, el sida y otras dolencias graves". (Asamblea Legislativa de la República de Costa Rica, 1998, p. 18.)

Ahora bien, no es la intención de esta síntesis historizar las trasformaciones institucionales que sufrió la legislación nacional en el pasado alrededor de la protección de la población menor de edad, ni tampoco, es estudiar la función histórica de las instituciones que las resguardan. Se hace esta aclaración porque la mayoría de las noticias tienen un lenguaje jurídico, por ende, lo que se quiere es partir de la fundamentación legal y vigente con la que se ha regulado desde 1998 hasta el presente los derechos de las personas menores de 18 años en Costa Rica. Por lo que es apropiado indagar, de qué manera, se intenta cumplir actualmente la protección del menor, desde su valor jurídico con los tipos de acusaciones o situaciones presentes en las noticias analizadas.

Las noticias pueden ayudar a reconstruir situaciones, constantemente se está haciendo un llamado a la población en varias de ellas, para analizar la violencia sexual no como un fenómeno reciente, ni aislado, ya que en los últimos dos años se ha vislumbrando una sociedad cada vez más agresiva en distintos términos, además del sexual, pero que en muchas ocasiones poseen cierta vinculación, por ejemplo, con el creciente consumo de drogas en la juventud, el peligro de las redes sociales y la inmigración en condiciones marginales u otras situaciones socioeconómicas que propicien la constante proliferación de la pobreza. De hecho, al respecto, el director general de la Fuerza Pública, Juan José Andrade, desde el primer día del 2014:

(...) hizo un llamado a los padres de familia para conocer el entorno en el que sus hijos se desenvuelven socialmente y en las redes sociales, área donde se pueden establecer relaciones con extraños 
cuyas consecuencias quedan muchas veces fuera del control parental (Solano, 1 de enero de 2014).

Dichas noticias y sus referentes han servido para contextualizar la situación por la que está pasando Costa Rica, actualmente, y que puede ser un fenómeno que se ha intensificado desde el año 2009, esto según lo visualizado por la mayoría de columnistas. Empero, se introducen temáticas de género, por ejemplo, con respecto al poco espacio de atención que se les da a las víctimas masculinas que fueron perpetrados sexualmente, se presentan algunas estadísticas que evidencian la situación vivida durante este proceso:

(...) el primer semestre del 2014, la Medicatura Forense llamó a 171 de los 336 hombres que denunciaron haber sufrido violaciones en el lustro anterior. A partir de esa muestra, se determinó que el $82 \%$ de los afectados eran menores de edad...106 eran menores de 12 años al momento de la agresión, y 35 tenían entre 12 y 18 años (Corrales, 16 de setiembre de 2014).

Por otro lado, e incluyendo al sexo femenino, se da un análisis comparativo desde la perspectiva de la desigualdad de género, lo cual revela lo siguiente:

Para el año 2013, según el Instituto Nacional de Estadística y Censos, se reportaron 473 nacimientos de madres menores de 15 años y 7 de padres menores de 15. En el rango de 15 a 19 años, 12.451 adolescentes se convirtieron en madres y 1.760 adolescentes varones en padres (Sánchez, 7 de junio del 2014).

Además de largometrajes relacionados con la homosexualidad adolescente y su rechazo social (Solano, 8 de marzo del 2015), hay un artículo con un lenguaje social, publicitario y entretenido, el cual llama a las personas telespectadoras a ver una narconovela llamada "Señorita pólvora", la cual tiene como sinopsis la historia de una niña que pierde a su padre en manos de un sicario, posteriormente, su madre la obliga a entrar en un concurso de belleza y se sexualiza desde corta edad, a raíz de esta situación, termina enredada con sicarios y traficantes de drogas; lo interesante para los fines de esta investigación, no es la novela en sí, sino, como bien podría ser la historia de cualquier menor de edad en Latinoamérica, como fueron los casos reales de “(...) Laura Zúñiga, exreina del estado de Sinaloa en el 2008 y la de María Susana Flores, dueña del título Mujer de Sinaloa 2012" (Miranda, 14 de marzo del 2014).
Las denuncias que le llegan al Patronato Nacional de la Infancia (PANI) son espeluznantes en crecimiento, se estima que en las últimas semanas del día 30 de agosto del 2014 “(...) las denuncias contra adultos que, al parecer, sostienen relaciones sexuales con menores, crecieron en un 30\%" (Recio, 30 de octubre del 2014), de igual manera se establece lo siguiente:

La entidad atribuye el aumento en los casos reportados a la campaña denominada Para el amor sí hay edad, que se difundió ampliamente por las redes sociales... El PANI lanzó una contra-campaña llamada "Para el amor si hay edad", para sensibilizar sobre las consecuencias de las relaciones entre menores y adultos... Rodolfo Meneses, abogado del Patronato, recordó que los adultos no pueden tener relaciones sexuales con menores de 15 años (Recio, 30 de octubre del 2014).

Posteriormente, se habla del artículo 159 en el Código Penal, el cual estipula el castigo con prisión de dos a seis años al que se aproveche de la edad para obtener algún tipo de contacto carnal. Asimismo, se critica la negligencia del sistema, ya que, según “(...) los datos del Patronato, el $88 \%$ de los embarazos de adolescentes son producto de una relación sexual con un hombre mayor de edad" (Recio, 30 de octubre del 2014). Se concluye que, actualmente, la mayoría de las 14.000 niñas y adolescentes que resultan embarazadas por año en Costa Rica terminan siendo madres producto de esa violencia sexual.

Asimismo, existen otros proyectos de asistencia social, como los del Hospital Max Peralta de Cartago, con los cuales se creó una Clínica dirigida a madres adolescentes, para asesorar temas como el cuidado del bebé, la planificación familiar, la salud sexual, entre otros. Aunque no se evidencian otro tipo de propuestas, sí se da el alza de voz por parte del Papa Francisco desde el Vaticano contra la pedofilia (Directriz a obispos, 6 de febrero del 2014). Ante tal situación, tiempo después durante el 13 de mayo del 2015, los diputados tuvieron una iniciativa en donde “(...) prohibirían por medio de una ley los matrimonios en los que al menos una de las dos personas involucradas sea menor de 18 años" (Sequeira-La Nación, 13 de marzo del 2015).

La situación más discutida en los periódicos se relaciona con los crecientes abusos sexuales y las torturas físicas que sufre la niñez dentro y fuera de Costa Rica, como en el caso de Nicaragua. De hecho, en el Anuario estadístico del Poder Judicial (2013) “(...) se detalló que el abuso sexual y la violación constituyen dos modalidades delic- 
tivas que vienen creciendo desde el 2009" (Arguedas, 25 de marzo del 2015).

La agresión a niños y niñas en Costa Rica se está duplicando, según casos registrados en 2014 por la policía, siendo la noticia del lunes 2 de febrero del 2015, donde se conoció la noticia del asesinato de un niño de solo dos años, aunque de las constantes referencias al caso, solo una nota periodística retomó a la madre nicaragüense del niño, menor de edad también, quien “(...) fue violada a los 13 años y quedó embarazada. Al ser agredida por su compañero, 25 años mayor que ella, emigró a Costa Rica, hasta donde él la persiguió" (Editorial, 17 de febrero del 2015).

\section{Los casos de violencia sexual infantil y ado- lescente}

Con lo que respecta a las 22 noticias sobre casos de violencia sexual, el lenguaje judicial es el predominante, de las 22 noticias, 21 hacían referencia a ese lenguaje, en menor medida estaban otros, como: el psicológico, el preventivo, el diplomático, el social y los usos testimoniales.

Los sospechosos, en su mayoría eran de clase obrera, y pocas veces aparecían profesionales, de hecho, si era el caso, se trataban de profesores de estudiantes afectados, solo hubo un caso de un asambleísta del Partido Acción Ciudadana (PAC) de la provincia de Guanacaste que violentó a un adolescente travestido, pero en la Ciudad de Natal, Brasil (Miranda, 20 de junio de 2014; Recio, 19 de junio de 2014; Araya, 20 de junio de 2014). El abuso y la violación sexual estudiados estuvieron muy vinculados y frecuentes en casi todos los casos, pocas veces se dieron referencias a privación de la libertad, maltratos o explotación sexual.

A continuación, se presentan algunas figuras según el Análisis del Discurso realizado con los artículos de casos:

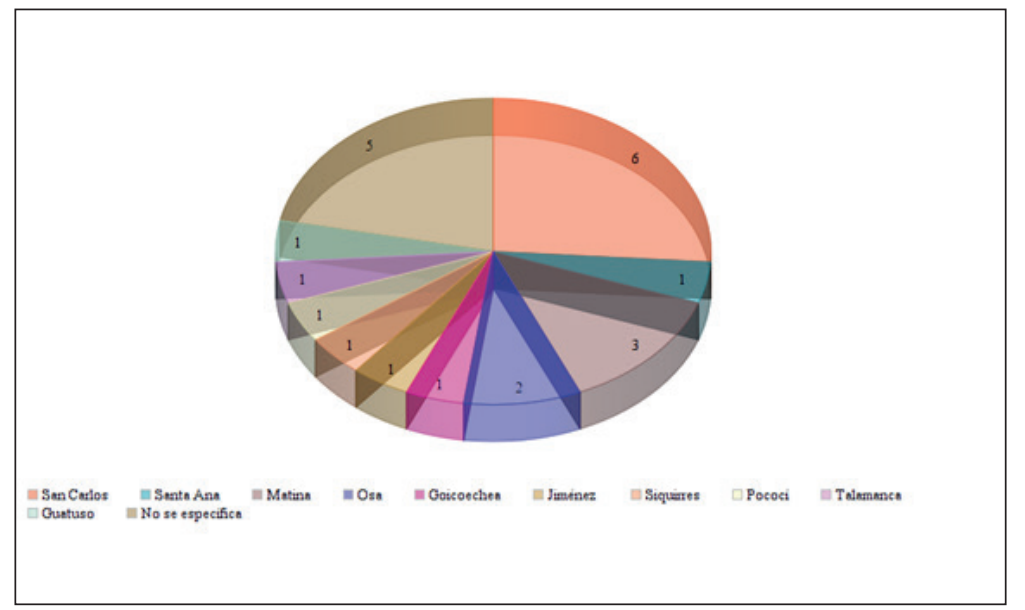

Figura 1. Ubicación del acto de violencia sexual según cantón (número y porcentaje). Fuente: elaboración propia, 2019.

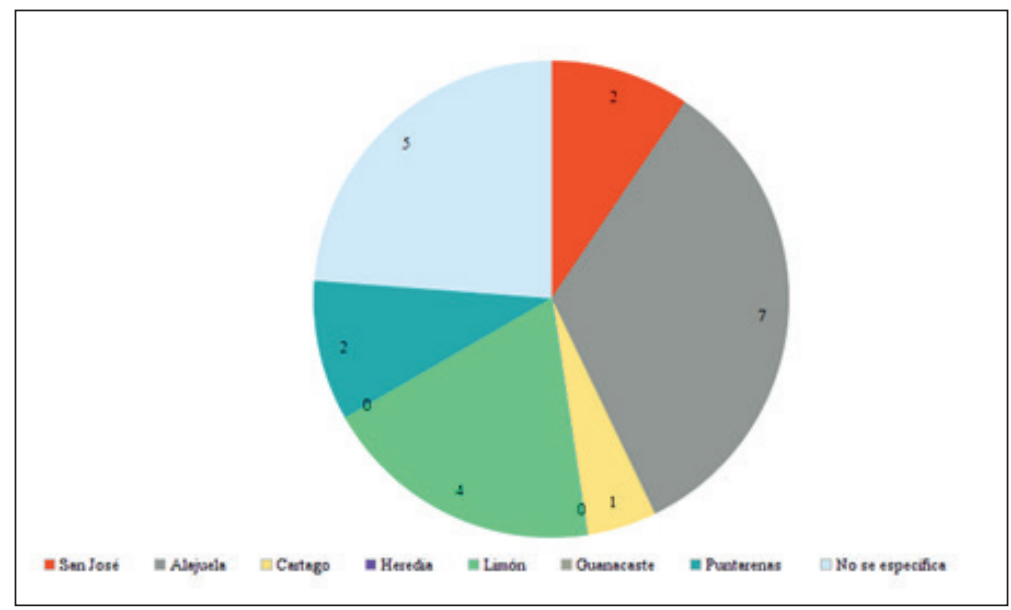

Figura 2. Ubicación del acto de violencia sexual según provincia (número y porcentaje). Fuente: elaboración propia, 2019. 
Con respecto a las figuras 1 y 2 se hace referencia a los acontecimientos de violencia sexual perpetrada contra menores de edad en tiempo y espacio, en donde se evidencia el poco control de este tipo de situaciones en las periferias del país, la provincia de Guanacaste es la más afectada por este fenómeno. En términos cantonales se puede apreciar como San Carlos (Alajuela) y Matina (Limón) se llevan el mayor peso del descontrol estatal, siendo estas dos zonas rurales unas de las más alejadas del poder central del país. Por su lado, Alajuela llama la atención al ser la única provincia del Valle Central que debido a su condición fronteriza se adentre en las dinámicas de proliferación de abusos y violaciones a menores, tal y como cualquier otra provincia periférica de Costa Rica. Asimismo, los cantones de San José parecen no ha- ber reportado tantos casos como sí sucedió en otras provincias, esto pese a ser la más poblada del país.

Con respecto a los datos etarios de las víctimas menores de edad durante el acto de la violación (figura 3 ) se puede apreciar que en la etapa de la preadolescencia y adolescencia femenina se dan con frecuencia las condiciones de mayor riesgo para las menores de edad, esto es representado por los tres primeros rangos gruesos de la pirámide graficada. En conjunto, se puede notar que las categorías que tuvieran la variable de la masculinidad tienen cifras bastante altas, lo cual revela la vulnerabilidad de este grupo, en cuanto a la condición de ser menor de edad; esto es aún más grave y repetitivo cuando el atributo femenino lo compone.

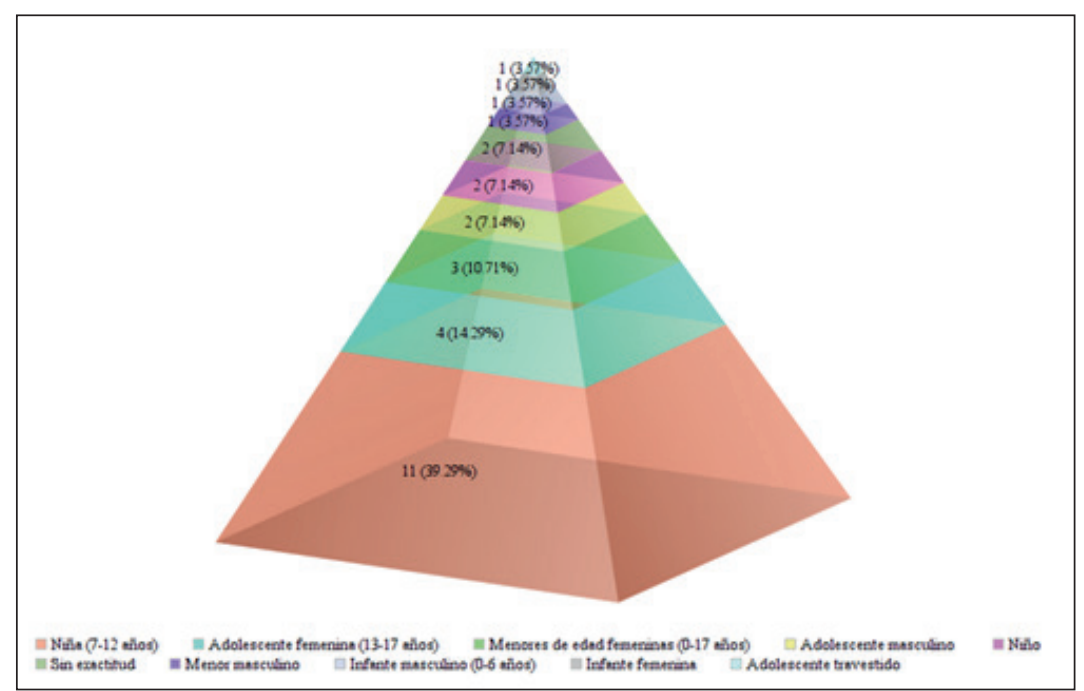

Figura 3. Etapa del desarrollo humano (etario) en que se encontraban las víctimas a la hora de la violación. Fuente: elaboración propia, 2019.

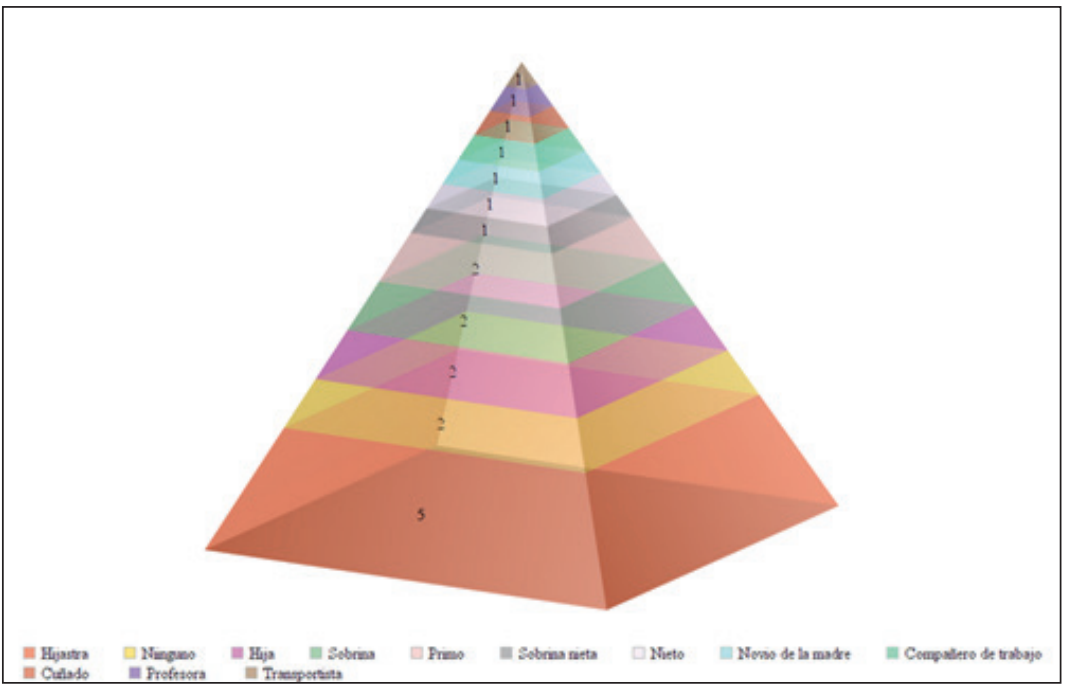

Figura 4. Vínculo de cada víctima con el sospecho. Fuente: elaboración propia, 2019. 
En la figura 4 se puede apreciar que básicamente los familiares cercanos y los profesionales que componen el ambiente cotidiano de un menor de edad son, principalmente, los sujetos sospechosos o impugnados de cometer el crimen de violación, esto revela lo poco sano que fue el espacio de interacción social del menor, posiblemente, se limitó su posición de comunicar lo que estaba sucediendo al estar en una estructura jerarquizada con posiciones de autoridad para los violadores, tanto familiar como escolar. Para comprobar lo anteriormente dicho, se puede apreciar que el nivel más alto de exposición se relaciona con las menores de edad en su condición de hijastras.

La nacionalidad del sospechoso (figura 5) no es explicitada en la mayoría de las noticias de este tipo del periódico La Nación, por lo que no se estaría al frente de un tema tratado con ese matiz de tinte xenofóbico en lo que respecta a este medio y los fenómenos de violencia sexual infantil. Sin embargo, el factor acerca de mencionar la nacionalidad de nicaragüense y costarricense siempre está presente en menor medida. xuales y los lugares donde se dan estos actos; Los Chiles (San Carlos) es el epicentro de la mayoría de los casos y el género femenino el más afectado. Finalmente, es evidente que el Código de la Niñez toma su accionar legal desde el Código de Familia y el Penal, todos juntos están tratando de cumplir la ley en el marco judicial, como lo revelan las noticias, pero, también se revela un descontento periodístico ante esto, pues no hay planes concretos en el ámbito nacional para evitar esto, más que publicidad y centros de lactancia para adolescentes. Por lo que es fácil concluir que los gobernantes no han ideado algún tipo de salida o estrategia frente a estas olas de violencia contra los sectores más jóvenes y vulnerables de Costa Rica, lo cual es un fenómeno latinoamericano y hasta global de agresión sexual.

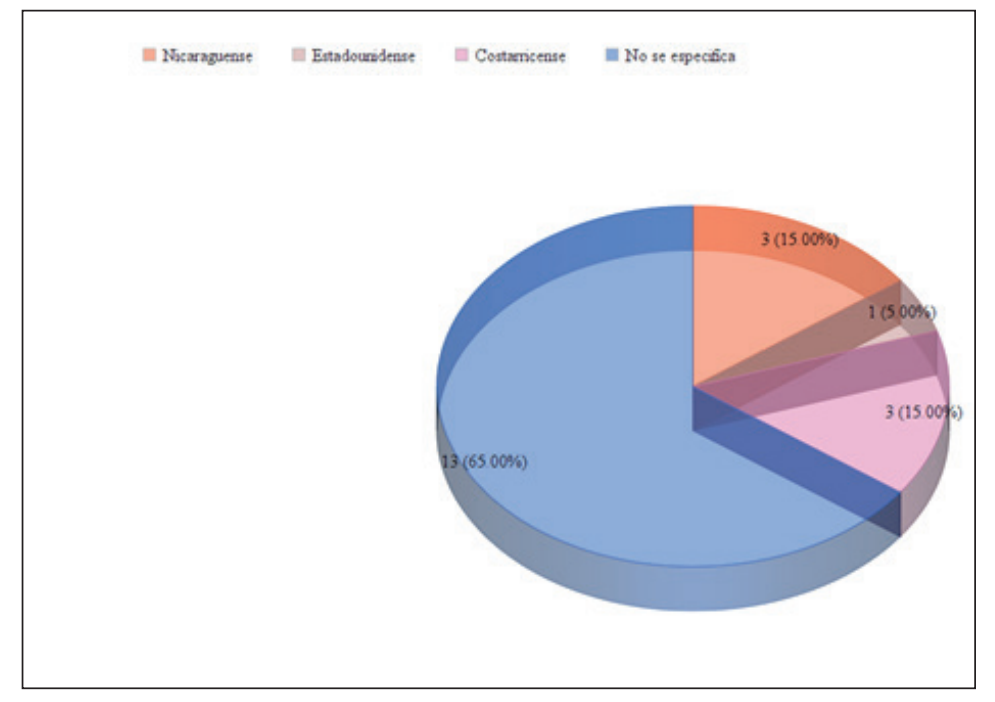

Figura 5. Veces que se da alusión a la nacionalidad del sospechoso en las 22 noticias de casos (número y porcentaje). Fuente: elaboración propia, 2019.

\section{Conclusiones}

El discurso periodístico pone en evidencia que el país está frente a una crisis en aumento de violencia sexual contra la población menor de edad. El punto clave de esta investigación es relacionar las estadísticas de los casos estudiados con las de carácter nacional, las cuales fueron vistas al inicio del trabajo como, por ejemplo, la presencia de un familiar o allegado en la mayoría de los abusos se-

\section{Referencias}

AFP. (2015, 13 de febrero). Las nuevas tecnologías pueden ser herramientas para tener a los niños bajo control. La Nación. http://www.nacion.com/tecnologia/gadgets/nuevas-tecnologias-herramienta-tener-control_0_1469253161.html

Araya, W. (2014, 20 de junio). Apresan a tico en Brasil por tener sexo con menor. La Nación. http://www. 
nacion.com/sucesos/poder-judicial/Apresan-tico-Brasil-tener-menor_0_1421657871.html

Arguedas, C. (2015, 25 de marzo). Artesano irá 22 años a la cárcel por violar a niño. La Nación. http://www. nacion.com/sucesos/juicios/Artesano-a n o s - c a r cel-violar-nino_0_1477452248.html

Asamblea Legislativa de la República de Costa Rica. (1998). Código de la Niñez y la Adolescencia.

Barrantes, A. (2015, 13 de febrero). 13.700 niñas se casaron con adultos en últimos 8 años. La Nación. http:// www.nacion.com/nacional/educacion/ninas-casaron-adultos-ultimos anos_0_1469453077.html

Bosque, D. (2014, 11 de enero). Sentencian a profesor por violar alumna en Bribrí. La Nación. http:// www.nacion.com/sucesos/crimenes-asaltos/ Condenan-profesor- violacion-alumna_0_1389861040.html

Castro, C. (2015, 6 de enero). El país del acoso. La Nación. http://www.nacion.com/opinion/foros/ pais-acoso_0_1461853807.html

Cerdas, D. (2014, 24 de octubre). Hombre sospechoso de violar a su hijastra de 9 años fue detenido en San Carlos. La Nación. http://www.nacion.com/sucesos/crimenes-asaltos/Hombre-sospechoso-detenido-San-Carlos_0_1447055410.html

Chacón, K. (2015, 25 de marzo). Autoridades detienen a sospechoso de violar a su sobrina de 4 años. La Nación. http://www.nacion.com/sucesos/poder-judicial/Autoridades detienen-sospechoso-violar- sobrina_0_1477452301.html

Chacón, K. (2015, 5 de abril). Agresión a niños en Costa Rica duplica casos registrados en 2014. La Nación. http://www.nacion.com/sucesos/crimenes-asaltos/Agresion-ninos-duplica-casos-registrados_0_1479652065.html

Chacón, K. (2015, 5 de abril). Los abusos sexuales son la principal agresión que sufren los niños. La Nación. http://www.nacion.com/sucesos/crimenes-asaltos/abusos-sexuales-principal-agresion-sufren_0_1479652066.html

Chacón, K. (2015, 5 de abril). Directora de HNN: La violencia está creando niños que serían abusadores. La Nación. http://www.nacion.com/sucesos/crimenes-asaltos/Ministra-Educacion-Huelga-obliga-desercion_0_1479652067.html
Chaves, K. (2014, 2 de noviembre). Seis hombres son violados cada mes en Costa Rica. La Nación. http://www.nacion.com/sucesos/seguridad/hombres-violados-mes-Costa-Rica_0_1448855176. $\mathrm{html}$

Chaves, K. (2014, 2 de noviembre). Me violaron dos hombres; me destruyeron la vida. La Nación. http://www.nacion.com/sucesos/seguridad/violaron-hombres-destruyeron-vida_0_1448855181. html

Chinchilla, E. (2014, 8 de junio). Policía acusado de abusar de menor solo estuvo preso 11 horas. La Nación. http://www.nacion.com/sucesos/seguridad/Policia-acusado-abusar-preso-horas_0_1425457467. html

Corrales, G. (2014, 16 de setiembre). Red de mentiras: El lado macabro de Internet. La Nación. http:// www.nacion.com/ocio/tv-radio/Red-mentiras-lado-macabro-Internet_0_1433456655.html

Delgado, D. (2014, 8 de marzo). Drogas, guaro y sexo se ofrecen en fiesta juvenil. La Nación. http://www. nacion.com/sucesos/narcotrafico/Drogas-guaro-ofrecen-fiesta-juvenil_0_1401059914.html

Delgado, D. (2014, 23 de setiembre). Error de diputados deja impune el abuso sexual. La Nación. http:// www.nacion.com/sucesos/seguridad/Error-diputados-impune-abuso-sexual_0_1440855951. html

Delgado, D. (2015, 8 de abril). Peón recrimina a jueces por condena pese a ser inocente. La Nación. http:// www.nacion.com/sucesos/juicios/Peon-recrimina-jueces-condena-inocente_0_1480251978. html

Directriz a obispos y otros funcionarios. (2015, 6 de febrero). Papa Francisco prohíbe encubrir pederastia en la Iglesia católica. La Nación. http://www.nacion.com/mundo/europa/Pontifice-prohibe-encubrir-pederastia-Iglesia_0_1468053205.html

Editorial. (2015, 17 de febrero). Nunca más. La Nación. http://www.nacion.com/opinion/editorial/maltrato_infantil-inmigracion-violencia_intrafamiliar_0_1470252979.html

Editorial. (2015, 13 de marzo). Breves País. La Nación http://www.nacion.com/nacional/Bre- 
ves-Pais_0_1474852526.html

EFE. (2014, 20 de octubre). Dos de cada tres niñas temen ser abusadas en el baño de sus centros educativos en Nicaragua. La Nación. http://www.nacion. com/mundo/centroamerica/temen-abusadas-centros-educativos-Nicaragua_0_1446255599.html

Gamboa, I. (2014, 4 de agosto). Con un grillo en la garganta. La Nación. http://www.nacion.com/opinion/foros/grillo-garganta_0_1430856907.html

Hernández, C. y Quesada, A. (2015, 5 de marzo). Peón agrícola violaba a hija quinceañera desde los 7 años. La Nación. http://www.nacion.com/sucesos/ seguridad/Peon-agricola-violaba-quinceaneraanos_0_1473452654.html

Hernández, C. (2014, 14 de marzo). Transportista de escuela abusó de tres menores. La Nación. http:// www.nacion.com/sucesos/seguridad/Transportista-escuela-abuso-menores_0_1475252505. html

Hernández, C. (2014, 8 de noviembre). Niña acusa a papá de múltiples violaciones. La Nación. http://www. nacion.com/sucesos/seguridad/Nina-acusa-papamultiples-violaciones_0_1450055017.html

Hernández, C. (2015, 25 de marzo). Padrastro encerró a niña un año en su casa para violarla. $\mathrm{La} \mathrm{Na}$ ción. http://www.nacion.com/sucesos/crimenes-asaltos/Padrastro-encerro-nina-casa-violarla_0_1477452271.html

Hernández, C. (2015, 26 de marzo). Madre de niña violada calla ante la Fiscalía. La Nación. http://www. nacion.com/sucesos/crimenes-asaltos/Madre-nina- violada-calla-Fiscalia_0_1477652248. html

Hernández, C. (2015, 28 de marzo). Padrastro sospechoso de violación queda libre por falta de pruebas. La Nación. http://www.nacion.com/sucesos/crimenes-asaltos/Padrastro-acusado-violar-quedo-libre_0_1478052217.html

Michael, M. (s. f.). Código de trabajo cumple 10 años de vigencia. UNICEF.

Miranda, Y. (2014, 14 de marzo). Señorita pólvora: La bella que se torna en bestia. La Nación. http:// www.nacion.com/ocio/tv-radio/Senorita-polvora-bella-torna-bestia_0_1475452462.html
Miranda, H. (2014, 20 de junio). Tico preso en Brasil podría ir hasta 10 años a cárcel. La Nación. http:// www.nacion.com/sucesos/seguridad/Tico-preso-Brasil-podria-carcel_0_1421857818.html

Montero, R. (2014, 30 de julio). Policía captura a menor por supuesto abuso a primo de dos años. La Nación. http://www.nacion.com/sucesos/ seguridad/Policia-captura-supuesto-abuso-primo_0_1429857252.html

Mora, A. (2015, 6 de febrero). Silencios y miedos de las mujeres. La Nación. http://www.nacion. com/opinion/foros/Silencios-miedos-mujeres_0_1468053187.html

Moya, R. (2014, 6 de noviembre). Supuesto violador fue arrestado en aeropuerto. La Nación. http://www. nacion.com/sucesos/seguridad/Supuesto-violador-detenido-aeropuerto_0_1449655045.html

Quesada, A. (2014, 16 de enero). Denuncian a abuelo por supuesto abuso de nieto. La Nación. http://www.nacion.com/sucesos/crimenes- asaltos/Denuncian-abuelo-supuesto-abuso-nieto_0_1390860937.html

Recio, P. (2014, 19 de junio). Costarricense detenido en Brasil por tener sexo con menor es asambleísta del PAC. La Nación. http://www.nacion.com/sucesos/seguridad/Costarricense-Brasil-asambleista-PAC-presidente_0_1421657953.html

Recio, P. (2014, 30 de agosto). Crecen denuncias por relaciones sexuales de adultos con menores. La Nación. http://www.nacion.com/nacional/Crecen-denuncias- relacion-adultos-menores_0_1436056425.html

Recio, P. (2015, 10 de marzo). Poder Judicial lanza App "Empodérate" para que niños denuncien violencia". La Nación. http://www.nacion.com/ tecnologia/apps/Poder-Judicial-aplicacion-denuncien-violencia_0_1474452675.html

Rodríguez, I. (2014, 12 de agosto). Sexo riesgoso tienta a jóvenes de Costa Rica. La Nación. http://www. nacion.com/vivir/bienestar/Sexo-riesgoso-tienta-jóvenes-pais_0_1432456764.html

Sánchez, A. (2014, 7 de junio). La vida de Adele: un torbellino de pasión se apodera de la Garbo. $\mathrm{La} \mathrm{Na}$ ción. http://www.nacion.com/ocio/cine/Adele-torbellino-pasion-apodera-Garbo_0_1419258081. html 
Sección de Sucesos. (2015, 20 de febrero). Tribunal de Pococí dictó 42 años de cárcel a peón por de siete violaciones. La Nación. http://www.nacion.com/ sucesos/Tribunal-Pococi-carcel-sospechoso-violaciones_0_1470853055.html

Sequeira, A. (2015, 12 de marzo). Avanza plan de ley para prohibir matrimonio con menores de 18 años. La Nación. http://www.nacion.com/ nacional/Avanza-prohibir-matrimonio-menores-anos_0_1474852601.html

Sequeira, A. (2015, 13 de marzo). Diputados prohibirían matrimonio de mayores con menores de edad. La Nación. http://www.nacion.com/nacional/ politica/Diputados-prohibirian-mayores-menores-edad_0_1475052517.html

Solano, H. (2014, 1 de enero). Lucha antidrogas y contra el matonismo son parte del operativo policial para la entrada a clases. La Nación. http://www.nacion. com/nacional/educacion/Fuerza-Publica-operativo-policial-entrada_0_1467053403.html

Solano, H. (2014, 18 de julio). Sujeto no se presentó a Juzgado y Policía lo captura por abusos deshonestos. La Nación. http://www.nacion.com/sucesos/ seguridad/Suejto-presento-Juzgado-policia-deshonestos_0_1427457428.html
Solano, M. (2015, 8 de marzo). La batalla contra la desigualdad. La Nación. http://www.nacion.com/opinion/foros/batalla-igualdad_0_1474052592.html

Tinoco, E. (2014, 19 de octubre). Trabajo forzoso y explotación sexual. La Nación. http://www.nacion. com/opinion/foros/Trabajo-forzoso-explotacion-sexual_0_1446055411.html

Vallejo, C. (2005). Representación de la violencia contra las mujeres en la prensa española (El País/ El Mundo) desde una perspectiva crítica de género. Un análisis crítico del discurso androcéntrico de los medios (tesis de doctorado). Universidad Pompeu Fabra.

Vargas, M. (2015, 12 de febrero). Nuevo sitio web ayuda a enseñar a los niños y adolescentes el uso adecuado de la tecnología. La Nación. http://www. nacion.com/vivir/ciencia/Nuevo-ensenar-adolescentes-adecuado-tecnologia_0_1469253105.html 